\title{
Evaluating Impact of an Emerging Big Health Data Platform: A Logic Model and Q- Methodology Approach
}

\author{
Dr. Justin Connolly \\ Dublin City University, Ireland \\ Prof. Anthony Staines \\ Dublin City University, Ireland \\ Prof. Regina Connolly \\ Dublin City University, Ireland \\ Dr. Andrew Boilson \\ Dublin City University, Ireland \\ Dr. Paul Davis \\ Dublin City University, Ireland \\ Dr. Dale Weston \\ Public Health England, United Kingdom \\ Dr. Natasha Bloodworth \\ Public Health England, United Kingdom
}

\begin{abstract}
Despite advances in technology and medical science, modern health-based projects are open to systemic failure due to many factors. These include I.T. developer's lack of awareness with regard to end-user needs, poor communication amongst all parties concerned and inappropriate or inadequate tests of the emerging system. Other issues may be external (e.g. political and legal) such as sharing of patient data and issues surrounding consent. The goal of this paper is to take a major health-based European model in current development and explore how it addresses the needs of four institutions in four different countries, and how it will meet their respective needs. The evaluation was designed within a Logic Model, and uses the Framework approach, and Q-Methodology to assess both impact and evaluation. Data will be collected through longitudinal semi-structured interviews and Q-scoring with principal stakeholders and developers at each stage of the project. This approach, recurring interviews with the same key players in the project, will help ensure that there is mutual understanding between I.T. developers and endusers of the system. The final system is meant to provide effective health-based decision support systems for policy makers.
\end{abstract}

Keywords: MIDAS Technology, Health-Based Projects, Decision Support Systems, Data Mining

JEL classification: 118

\section{Introduction}

Because of the complex nature of healthcare, ICT projects have extra challenges, and raise issues which are not always evident in other fields of the discipline. As Abouzahra (2011, p. 46) states: "IT projects in the healthcare sector have many differentiating characteristics over other types of projects. These characteristics rise 
from the sensitive nature of the healthcare environment as well as the diversity in user groups and IT systems usually installed in hospitals".

Awareness of these issues in conjunction with the ability to evaluate and address them is therefore central to successful adoption of technology-based health systems. This paper describes an emerging big data health platform and an evaluation of the factors that have potential to influence its success. Q-Methodology and a Logic Model framework are employed to provide an evaluation of the emerging platform and assess its overall impact. The paper begins by showing how the different characteristics of healthcare environment affect the success or failure of IT projects. It then describes the MIDAS project, the methodology that is currently being employed to increase its adoption and a brief description of the technical issues involved.

\section{Failure of Large Research Projects}

In order for projects to be successful, lessons must be learned from the past with regard to previous technologically-driven healthcare projects. The reasons for the failures of many large IT projects in the healthcare sector are complex, and influenced by internal, external or a combined factors. Research by Lu et al. (2010) suggests that internal factors in project failure involve variables strongly related to project management processes and project team dynamics. They posit that such internal issues are responsible for and are far more influential in project failure than external issues. In healthcare projects such issues may be political or legal such as sharing of patient data and issues surrounding consent. Key factors include poor communication or misunderstanding between developers and end-users of the system. In certain cases, users may become confused between their wants and actual needs and their grasp of data analysis techniques may lack the sophistication required to enable best use of the available data. Objectives may be impractical or unrealistic and impossible to achieve, particularly given strict time and financial constraints. Once the system is implemented there may follow inappropriate or inadequate testing of the emerging system. Pinto et al. (1990) consider the main factors of project failure to include an incomplete or inaccurate vision of project objectives, a failure to correctly identify and include the involvement of stakeholders, and communication and risk management issues. Such factors can have a cascade effect in that changes to the project may increase, customers are dissatisfied with outcomes, the quality of deliverables is poor and it may cause poor morale amongst developers. Furthermore, extended schedules inevitably lead to increased project costs.

Due to the multiplicity of such factors and differing stakeholder pressures and contexts, there is a greater recognition of the importance of evaluating impact as a health system project evolves. These evaluations must take into consideration the resulting impact(s) identified in that evaluation and not merely provide a review or account of what happened. Stakeholders, shareholders and those funding largescale projects need to see measures of program effectiveness as well as progress and descriptive reports of activities (O'Neill, 1998). Conversely, evaluation should consider the impact of programs and not merely the results from those programs. Impact represents results or accomplishments at a higher level. Ideally, the results of a program should ultimately change people's behaviour, attitudes, or bring benefit to society in some other form (Diem, 1997). Therefore, impact refers to implications of a given output, program, or project beyond the immediate intended outcomes. In particular, there is an emphasis on the broader long-term effects beyond the project itself. In effect, the ramifications of impacts resulting from this project will extend to 
society and influence decisions in health-based policy making, sharing of health data and governance best practice.

\section{The MIDAS Project}

Healthcare systems (Kruse et al., 2016) store patient data on large database systems where the data is heterogeneous and siloed. However, sharing of patient data at regional, national and cross-national level is increasingly needed to support integrated care, and provides an opportunity to better understand, prevent and predict potential health and healthcare problems. Furthermore, it is believed that availability of such data will help to reduce costs to healthcare providers. Many healthcare systems worldwide (Hicks, 2017) are adopting an "outcomes based healthcare" approach. Using data from a variety of sources, healthcare providers have the potential to identify which treatment works best for individual cases and at a more demographic level. Such healthcare systems aim to help policy makers within the medical field and at government level to improve the quality of patient health care.

The Meaningful Integration of Data, Analytics and Services (MIDAS) Project is a European-centered healthcare project. Its main purpose is to optimize use of current healthcare data to better inform public policy and improve healthcare and social well-being outcomes across Europe via a unified big data platform. It intends to achieve this by integrating patient data from various European health authorities where individual data will be collated and analyzed using various bespoke applications, modeling and visualization tools. Data will also be gathered via social media. The data will be analyzed on the MIDAS platform. It is expected that this pioneering healthcare platform will enable and provide tools for end-users, in particular policy makers, to benchmark, simulate and predict outcomes that will influence future healthcare policy decisions at both regional, national and European level. There are four use cases involved in this project, based in Northern Ireland, the Republic of Ireland, Finland and the Basque Country. Currently, European healthcare systems generate considerable data on a day-to-day basis. Such data includes patient prescriptions, patient care, hospital discharge records, waiting lists, data on blood-sugar levels, cardiac-related issues, etc. However, the data is localized and external access is difficult, thus limiting our understanding of health-based issues. This technical platform will not only provide critical insights into the health of different populations, but will enable policy makers to design and develop evidence-based preventative strategies which will address health and social care challenges at a wider level than is currently possible. Data analysis will enable policy makers to explore health trends, identify correlations and patterns amongst the general population and test various theories (e.g. diet patterns and obesity amongst particular regions according to age group and gender).

Overall, the MIDAS system is expected to be user-friendly and provide access to data analytics and visualization tools without the need for data-science expertise. It is also anticipated that there will also be a focus on simple, routine analytics with an element of prediction. A current problem with data systems is the lack of available analytics and tools for data mining. It is believed that the MIDAS tool will highlight gaps in the system and facilitate data system linkage to answer additional research questions and enable analytics and work that previously wasn't possible. At its core level, the MIDAS platform will utilize Analytics Engines XDP which operates on three core principles: (i) it facilitates access to the data from a singular location without the need for replication; (ii) the data is analyzed once and the process of analysis can be reused as the data is updated; (iii) data sharing and analysis is feasible through 
repeatable processes (Analytics Engines). The MIDAS technical teams will install this form of data analytics in the four European healthcare systems for data integration, analytics and visualization. However, stakeholder understanding of analytics and other core technical issues is paramount to successful outcomes. Good data mining techniques and optimum use of decision-support systems is dependent on individual competence in using the technology presented. One key technical challenge is in making the system a very useable platform for end users not highly experienced in data analysis techniques. This issue has to be balanced against a need to ensure that the system produces health-based reports that are easy to generate, but provide output that is meaningful and accurate. The system will also support timeseries analysis and projection analysis to provide accurate forecasting of potential health issues based on the health data available at regional, national and crossborder level. An expected outcome from the MIDAS system will be the use of predictive modeling as an analytical tool, which, in turn, will help to prevent rather than treat certain conditions. This will also influence future health education projects. It is hoped to connect existing datasets and reduce fragmentation in order that the true value of combined datasets can be unlocked.

\section{Methodology}

As discussed in the previous section, many large-scale health system projects fail due to a variety of internal and external issues. In order to ensure a successful outcome for the MIDAS project, it was decided to undertake a thorough and in-depth evaluation and impact assessment methodology. The initial evaluation was designed as a multi-pronged approach involving the development of a Logic Model, qualitative interviews with stakeholders and developers, and the use of QMethodology to assess both impact and evaluation. Data was collected in the first round of interviews through longitudinal semi-structured interviews. These were transcribed and coded using the Framework Approach (Ritchie et al., 2003). This approach - recurring interviews with the same interviewees (stakeholders and developers involved in the project) - helps ensure that there is mutual understanding between I.T. developers and the end-users of the system at key stages of the project and any inconsistencies can be eliminated. A report of key findings from the coded transcriptions is then completed. In all, interviews will be conducted four times at key points throughout the lifetime of the project.

As previously stated, the logic model was used as a framework for development of the MIDAS evaluation plan. It links outcomes (both short- and long-term) with program activities/processes and the theoretical assumptions/principles of the program. It is defined as 'a systematic and visual way to present and share your understanding of the relationships among the resources you have to operate your program, the activities you plan, and the changes or results you hope to achieve' (Kellogg, 2004, p.1). At its most basic level, a logic model is a tool used in the planning, evaluation and systematic development of a project. Figure 1 (below) illustrates the dynamics involved in using the logical model. Stages 1 and 2 relate to planned work; stages 3 to 5 relate to intended results. 
Figure 1

The Basic Logic Model

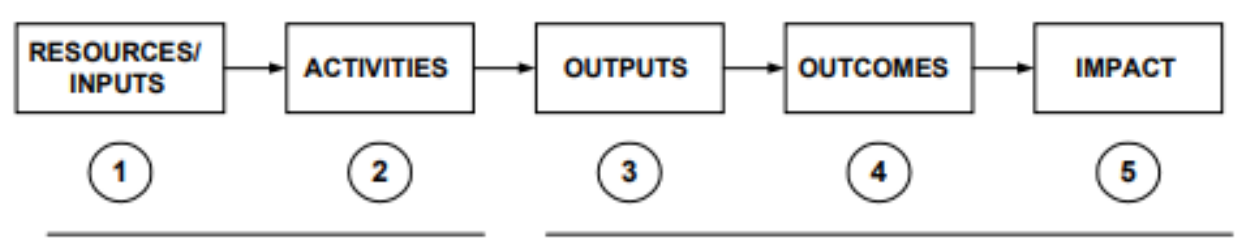

Source: W.K. Kellogg Foundation, Logic Model Development Guide, p.3.

\section{Interviews \& Participant Sample}

Interviewees were selected from four European health institutions in Finland, the Basque region, Northern Ireland and the Irish Republic. The objective was to conduct semi-structured interviews separately with both developers and stakeholders involved in the project. These interviews take place four times over the duration of the project. The objective was that a minimum of two stakeholders per region were to be interviewed, (one technical person, and one policy maker. Questions were submitted to the interviewees prior to the interviews to enable them prepare adequate responses and raise questions if clarity was considered necessary. This was of particular value to those whose first language was not English. Questions related to end-user's understanding of the developing system, concerns and their perceived needs/requirements of the system. Each interview was recorded and transcribed. Following transcription, the interviews were sent to the interviewees to confirm accuracy and to enable additional information to be provided that may not have been mentioned during the recorded interview. The interviews were then coded. A report was generated based on the key findings from the coded material for developers and members of the MIDAS consortium to consider.

\section{Q Methodology}

Q Methodology takes a subjective approach to data analysis and is a combination of both qualitative and quantitative research methods. It is principally used in the fields of psychology and the social sciences and it is particularly effective in identifying attitudes, perceptions, feelings, and values. Developed by William Stephenson (a psychologist) in the 1930s (McKeown et al., 1988), it is used in research settings where individual perspectives on a topic can be analyzed for consistency or deviation over time. Essentially, Q Methodology derives from factor analysis. However, whilst standard factor analysis uses the "R method" to find correlation between variables from a data sample, $Q$ is used to identify correlations between subjects from a sample of variables. It does this through the use of ranking. The statements used in the $Q$ sample are derived from and represent a "concourse" which is the set or sum of statements pertaining to the topic being investigated. These statements relate to those used in interviewing the various developers and shareholders in the interview cycle. This particular research method will also help to identify if and how the interviewees rankings change over time based on individual attitudes and beliefs. In-depth follow-up interviews will involve gathering information based on the responses provided during the $Q$-sort or ranking of variables. 


\section{Conclusion}

This paper has outlined the use of a logic model and Q Method in the context of a large-scale cross-national big data platform. It points to the value of such an evaluation approach and its potential to increase successful adoption of the final technical platform. Healthcare ICT projects are intrinsically complex, and without careful planning and implementation, they are likely to fail. Beyond the technical issues and stakeholder requirements involved, there are legal and political issues to be considered. Development of the MIDAS project has been, and continues to be, an ongoing process of evaluating outcomes and identifying potential impacts to reduce the possibility of critical issues emerging. Applying a systematic and rigorous approach to each stage of the developmental process will help to ensure the project's success using proven research methods. Project success is further supported through regular communication between technical developers and the stakeholders or end users of the system. It is expected that the final system will enable better data mining techniques through use of new tools developed specifically for patient data analysis and decision-making by policy makers. It also demonstrates the effectiveness of a logic model and $Q$ Method approach in evaluating impact, thereby increasing the alignment of the technical system and its functionality with the requirements of the end user, which will increase potential adoption of the system.

\section{References}

1. Abouzahra, M. (2011), "Causes of failure in Healthcare IT projects", in proceedings of the 3rd International Conference on Advanced Management Science, Vol. 19, IACSIT Press, Singapore, pp. 46-50.

2. Analytics Engines, "Innovation through industrialisation", available at: http://www.analyticsengines.com/analytics-engines-xdp/ (22 March 2018)

3. Diem, K.G. (1997), Measuring impact of education programs, Rutgers University, New Brunswick, NJ.

4. Hicks, N. (2017), "Delivering an Outcomes-based NHS: Creating the Right Conditions", available at: https://www.ohe.org/publications/delivering-outcomes-based-nhscreating-right-conditions (25 March 2018)

5. Kellogg, W. K. (2004), Using logic models to bring together planning, evaluation and action: Logic model development guide, Kellog Foundation, Battle Creek, MI.

6. Kruse, C. S., Goswamy, R., Raval, Y., Marawi, S. (2016), "Challenges and Opportunities of Big Data in Health Care: A Systematic Review", JMIR Medical Informatics, Vol. 4, No. 4.

7. Lu, X., Liu, H., Ye, W. (2010), "Analysis failure factors for small \& medium software projects based on PLS method", in proceedings of the 2nd IEEE International Conference on Information Management and Engineering (ICIME), IEEE, Chengdu, China, pp. 676-680.

8. McKeown, B., Thomas, D. B. (1988), Q Methodology, Sage, Newbury Park, CA.

9. O'Neill, B. (1998), "Money talks: Documenting the economic impact of Extension personal finance programs", available at: https://www.joe.org/joe/1998october/a2.php (22 March 2018)

10. Pinto, J. K., Mandel, S. J. (1990), "The Causes of Project Failure", IEEE Transactions on Engineering Management, Vol. 37, No. 4, pp. 269-276.

11. Ritchie, J., Lewis, J. (2003), Qualitative research practice: a guide for social science students and researchers, Sage, London. 


\section{About the authors}

Dr. Justin Connolly completed his PhD at Dublin City University and currently works as Assistant Researcher in the School of Nursing and Human Sciences on the European H2020 MIDAS project. His background and interests are in technology, sociology and health-based systems. The author can be contacted at justin.connolly@dcu.ie.

Professor Anthony Staines is Professor of Public Health at Dublin City University. He started out as a neonatal paediatrician, moved into public health, and academic epidemiology. His interests lie in the area of child public health, health information systems, the social costs of illness, blood transfusion policy, including stock management, multiple myeloma, and the financing of primary care. The author can be contacted at anthony.staines@dcu.ie.

Professor Regina Connolly specialises in Innovation Adoption at Dublin City University. A regular speaker both in Ireland and internationally, she has presented on Connected Health Business Models at the European Parliament. She has significant expertise in healthcare technology impact assessment as well as e-Health business model development. The author can be contacted at regina.connolly@dcu.ie.

Dr. Paul Davis is Head of the Management Group (School) in Faculty of Business in DCU. He has previously been seconded to the Health Service Executive ICT Strategy Unit, advising on procurement strategy and market engagement. The author can be contacted at paul.davis@dcu.ie.

Dr. Andrew Boilson is a Post-Doctoral Researcher on the MIDAS project in the School of Nursing and Human Sciences at Dublin City University. His background is in psychology, research and e-health. The author can be contacted at andrew.boilson2@mail.dcu.ie.

Dr. Dale Weston is a Research Fellow working in the Emergency Response Department Science \& Technology Behavioural Science team at Public Health England. His interests lie at the intersection of Social Psychology and Health Psychology with a particular interest in applying theories and research within the context of public health. The author can be contacted at dale.weston@phe.gov.uk.

Dr. Natasha Bloodworth completed her BSC and MSc degrees at the University of Plymouth. She also holds a PhD in Psychology from the University of Exeter. She currently works as a Research Fellow at Public Health England. The author can be contacted at natasha.bloodworth@phe.gov.uk. 